\title{
THE USE OF FRACTAL GEOMETRY FOR THE ASSESSMENT OF THE DIVERSIFICATION OF MACRO-PORES IN CONCRETE
}

\author{
JANUSZ KONKOL AND GRZEGORZ PROKOPSKI \\ Department of Materials Engineering and Technology of Building, Rzeszów University of Technology, \\ Powstańców Warszawy 6, 35-959 Rzeszów, Poland \\ e-mail: janusz.konkol@prz.edu.pl,grzeprok@prz.edu.pl \\ (Accepted March 31, 2011.)
}

\begin{abstract}
The article presents the results of examination of the fractal dimension $D$ of macro-pores in concrete. The concretes were made from coarse basalt aggregate. Fractal examinations showed that the fractal dimension $D$ of macro-pores in concrete was different, depending on the water-cement ratio $W / C$. The performed examination of macro-pores in concretes has shown the existence of a statistically significant correlation between the fractal dimension $D_{B C}$, as determined by the box method, the relative number of pore crosssections $N_{A}$ (or the number of pores $N$ ) and the total air content $A$, as well as between the fractal dimension $D_{B C}$, the relative number of pore cross-sections $N_{A}$ and the water-cement ratio $W / C$.
\end{abstract}

Keywords: concrete, fractal geometry, stereology.

\section{INTRODUCTION}

Pores are an inherent constituent of concretes. The cause of their formation is different: they either form during preparation of a concrete mixture or result from hydration reactions. The influence of pores on both the mechanical and physical characteristics of concrete is indisputable. The structural pores discussed and analyzed in this work are the least wanted kind of pores. Size, shape, and number of such pores depend to a large degree on workability and consistency of the concrete mix. For regular concretes these parameters are strictly correlated with water-cement W/C ratio.

Various methods of determining the air content of concrete, either of the concrete mix or of the set concrete, are used. Some of the methods to assess porosity, in addition to the determination of the total porosity, enable also the assessment of the size of pores, their shape and manner of distribution (Gregg and Sing, 1982; Cook and Hover, 1999; Diamond, 2000; Stroeven and Guo, 2006). A method enabling the determination of the air content of a fresh, compacted concrete mix is the pressure method; however, the porosity determined by this method constitutes only some part of the total porosity.

The methods enabling the assessment of the porosity of set concrete include: the mercury intrusion porosimetry (MIP) method (Cook and Hover, 1999;
Diamond, 2000), nitrogen (Gregg and Sing, 1982) and water vapour sorption methods (Daian, 1988), the stereological measurement method using an image analyzer, and the method using gallium (Ga intrusion method) (Tanaka and Kurumisawa, 2002); however, only in the case of the stereological measurement method using an image analyzer, the actual distribution of pores within concrete and their shape can be assessed.

The achievements of today's science and technology allow us to take advantage of ever newer tools and methods of description of the complex structures which are pores. A parameter that well characterizes the structure of pores is the fractal dimension $D$, as defined by Mandelbrot (1977). The total volume of pores may be the same for two materials with a different distribution of pores; a help with differenttiating such materials can be the fractal dimension, being dependent on both the number of pores and their size and shape.

One of the methods of determining the fractal dimension, $D$, is the box method, which is a variant of the cover method. According to this method, the area to be analyzed is covered with squares of a certain side $s$ and then the squares intersected by pore edges are counted. The counting is repeated several times, while changing the length of the square side $s$. The fractal dimension $D$ is determined from the slope of the straight line in the bi-logarithmic diagram of 
the dependence of logarithm of the number of boxes $N(\log N(s))$, as counted at a given step $s$, on the logarithm of box dimension $\log (s)-$ Fig. 1 .

First studies confirming the fractal character of the surface of hydrated cement paste were carried out by Winslow (1985), and then these studies spread fairly widely. Presently, in the examination of concretes, the fractal dimension is used for the description of the degree of complication of the surface scratching of cement materials (Carpinteri et al., 1997; Chiaia et al., 1998; Ringot and Bascoul, 2001, Yan et al., 2002), the characterization of the fracture surfaces of cement materials (Winslow, 1985; Carpinteri et al., 1999; Carpinteri and Spagnoli, 2004; Konkol and Prokopski, 2004; Prokopski and Konkol, 2005; Carpinteri et al., 2010), the assessment of the fractal dimension of aggregate grains (Li et al., 1993), and in the analysis of pores occurring in cement paste (Lange et al., 1994; Ji et al., 1997; Hu and Stroeven, 2003).

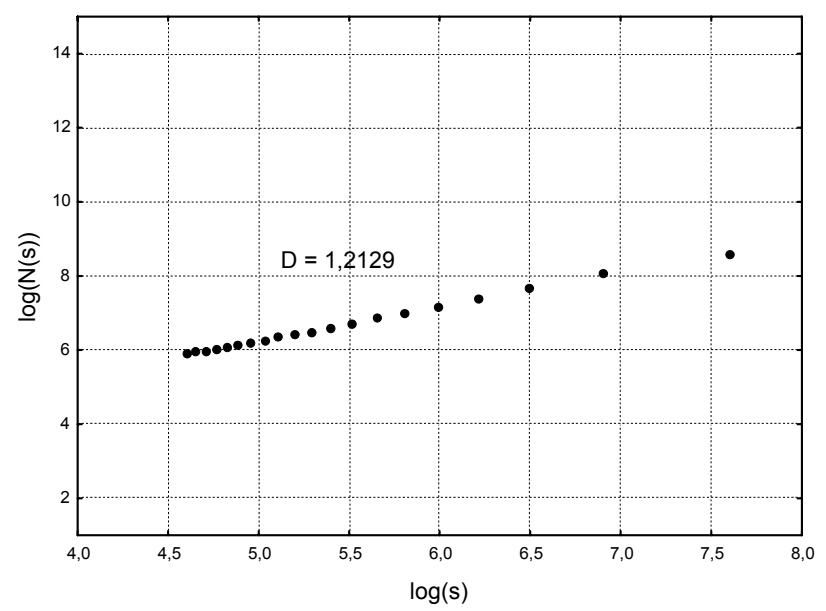

Fig. 1. Examples of the graphs of dependence of log $N(s)$ on $\log (s)$.

\section{METHODS}

The first methods of the porosity determination were those of sorption and porosimetry. However, the mercury intrusion porosimetry and sorption methods not always can be sufficient. They allow the determination of the characteristics of small-size pores. It appears reasonable and purposeful to use other methods enabling a full picture of pores in a given material to be obtained. By making the analysis of the distribution of pores in cement materials, Diamond (2000) has shown that the mercury intrusion porosimetry method is not sufficient. He also compared results obtained using the mercury intrusion porosimetry method and the image analysis method.

The examination of nitrogen sorption consists of the determination of the specific area by the singlepoint or multiple-point method and the determination of the constants of the Langmuir and BET adsorption isotherm equation (Gregg and Sing, 1982). The examination of water vapour sorption, on the other hand, can be performed by the gravimetric method, which involves placing specimens in dessicators with saturated solutions of appropriately selected salts until the state of thermodynamic equilibrium is reached (Daian, 1988). It has been found (Daian, 1988), however, that the use of either method may lead to obtaining different results, and the adopted model for the course of the phenomenon does not always reflect the actual one. Good agreement between the actual and theoretical isotherms was obtained within the scope of small-size pores. In the case of larger-size pores, better agreement was obtained using the mercury intrusion porosimetry method. The occurred differences might result from the destruction of the smallest (macro- and micro-) pores by mercury forced into the specimen (Daian, 1988).

A solution complementing the above-mentioned methods and, at the same time, the only method enabling the observation of the pores, can be stereological measurements using, for example, an image analyzer (ASTM C 457 - 98). Such measurements are used, among others, for the assessment of the freeze resistance of building materials, while the pore characteristics are determined using the linear traverse method or the punctual method (ASTM C 457 - 98; Aligizaki and Cady, 1999; Elsen, 2001). The results of extensive comparative studies have confirmed that the obtained results on overall porosity may vary, depending on the method applied (Elsen, 2001).

The proper formation of the freeze-resistant structure in the design and execution of a construction is of particular importance during its operation. A crucial role play the amount of water contained in the concrete, capable of being frozen, as well as the volume and distribution of air pores. Assuring the proper freeze resistance involves the provision of maximal water-tightness, i.e., an impermeable structure free from water capable of being frozen, and producing an appropriate system of air pores not able to be completely filled with water, which, at the same time, will contribute to an improvement in the thermal properties of the material. This can be achieved through: 
using special treatments (such as the modification of the hydration process, or aeration), special setting up of concrete mix composition, using low water-cement $(\mathrm{W} / \mathrm{C})$ ratios, additions and admixtures (such as plasticizers, silica dusts) and the appropriate mixing time and consolidation and conditioning method.

A parameter that relates the porosity structure of aerated concrete to its freeze resistance is the pore spacing factor, $\bar{L}$, defined by Powers (1949) as the average distance of an arbitrary point in the cement paste from the edge of the nearest air bubble. Its value for a concrete of satisfactory freeze resistance should be below $0.2 \mathrm{~mm}$ for severe environmental conditions, and below 0.25 in the case of moderate environmental conditions.

This coefficient is defined on the basis of stereological relationships (ASTM C 457), according to the expressions below:

$\bar{L}=\frac{p}{400 \cdot n}$, when $: \frac{p}{A} \leq 4.342$

and

$\bar{L}=\frac{3}{\alpha}\left[1.4 \cdot\left(\frac{p}{A}+1\right)^{1 / 3}-1\right]$, when $\frac{p}{A}>4.342$

where:

$p$ - paste content in $\%$,

$A$ - air content in \%,

$n$ - void frequency,

$\alpha$ - pore specific surface.
The specific surface, $\alpha$, is the total area of a given phase related to the unit volume of that phase. This quantity is regarded as an universal index of particle dispersion, regardless the shape of the particles. In practice, two methods of measuring the specific surface are used, namely: the random secant method in combination with the point-count method and the random secant method in combination with the linear-traverse method. For example, in the case of the random secant method in combination with the linear-traverse method, the specific surface, $\alpha$, is determined from Eq. 2 and Fig. 2 in the following manner (ASTM C 457):

$$
\alpha=\frac{4}{\bar{l}} \text {, where } \bar{l}=\frac{T_{a}}{N},
$$

where:

$\bar{l}$ - average chord length,

$T_{a}$ - traverse length through air,

$N$ - total number of air voids intersected (number of chords),

$l_{i}$ - length of the $i$-th chord.

The estimators of stereological parameters describing the spatial structure of a given material can be defined in a relatively straightforward way on the flat cross-sections or projections of this structure. A basis for such a description is the computer-processed and analyzed image. Stereology enables relationships to be established between the three-dimensional space parameters and the parameters of lower-dimension spaces.

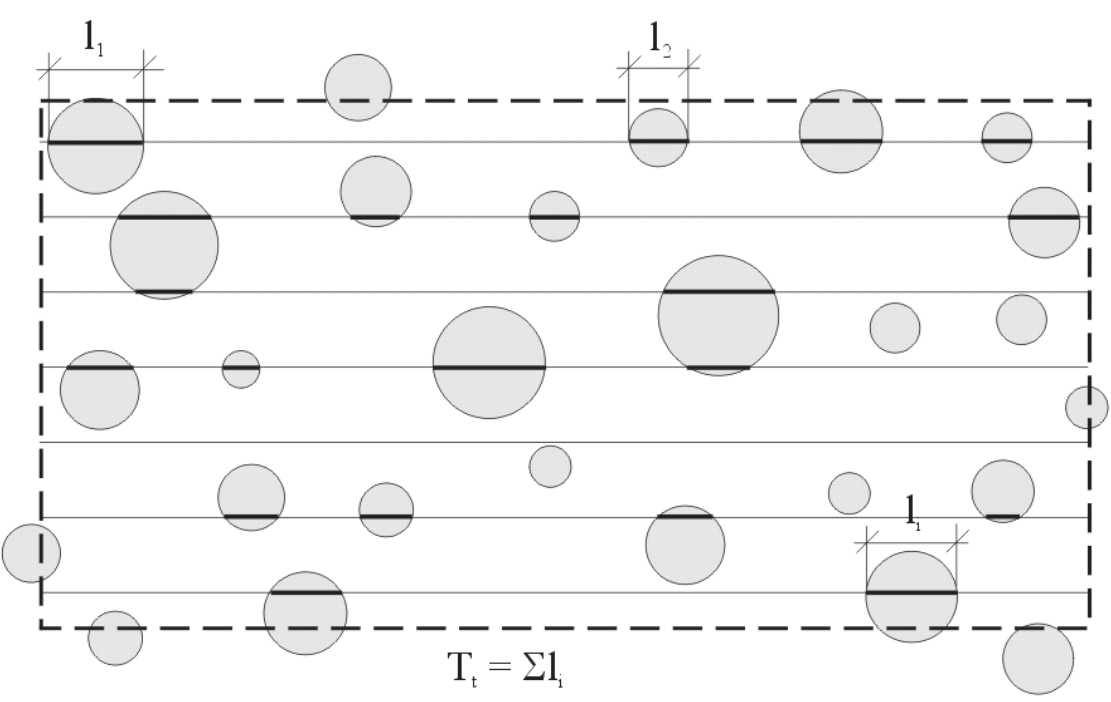

Fig. 2. Schematic diagram for the determination of the specific surface, $\alpha$, using the secant method in combination with the linear-traverse method. 
The basic parameters determined by the stereological methods include:

a) the total air content, $A$, defined as the sum of pore cross-section areas on a flat concrete specimen, related to the specimen area, $A_{Z}$. This value is most often given in percents;

b) the pore specific surface, $\alpha$, described by Relationship (2) and Fig. 2;

c) the relative pore number, $N_{A}$, defined as the ratio of the number of pores $N$ on the flat specimen image examined to the area of this image $A_{Z}$, see Eq. 3 in the following;

d) average chord length, $\bar{l}$, calculated as the ratio of the sum of chord lengths to the length of all secants.

Important problems when making the stereological analysis of pores in concrete are: the method of specimen preparation, the method of acquiring a computerized image of appropriate resolution and the computer image analysis.

Two methods of specimen preparation and computer image acquisition are compared below. The analysis of pores in concretes was carried out using the computer program FRAKTAL_Stereolog ${ }^{1}$ and the image analyzer Image Pro Plus 4.1 (Załocha, 2002). The method of interpreting the locations of pores by the FRAKTAL_Stereolog program is shown in Fig. 3.

Fig. 3 shows variation in the greyscale along the length of a sample line passed through the real image of a basalt aggregate concrete specimen. The line at height 210 separates the places recognized as pores (above line 210) and the places of occurrence of cement paste and basalt coarse aggregate grains (below line 210). The employed method of identifying the structure elements enables also the recognition of locations of occurrence of coarse aggregate grains; the greyscale below 70. A detailed method of preparing the concrete specimen surface for stereological examination is described in work Konkol and Prokopski (2004).

A preliminary examination was carried out, consisting in the analysis of the effect of scanning resolution (from 100 to $1200 \mathrm{dpi}$ ) and the adopted greyscale tolerance (the lower threshold from 160 to 250). As an optimal solution, a resolution of $400 \mathrm{dpi}$ and a lower greyscale threshold of 210 was chosen.

The accuracy for the analysis performed on concrete specimens using the FRAKTAL_Stereolog computer

1 Konkol J.: FRAKTAL_Stereolog. A computer program. program and a scanner (with an image resolution of $400 \mathrm{dpi}$ ) was $6.36 \mu \mathrm{m}$ (the magnitude corresponding to 1 pixel), while it was $2.76 \mu \mathrm{m}$ using the Image Pro Plus 4.1 image analyzer operating with a stereoscopic microscope.

Below, examples of results obtained for ordinary basalt aggregate concrete of $W / C=0.44$ (Series $8^{2}$ ) and of $W / C=0.58$ (Series $9^{2}$ ) are presented. A detailed composition of these concretes, as well as the relationship between the porosity and the concrete fracture surface morphology is given in work Konkol and Prokopski (2004).
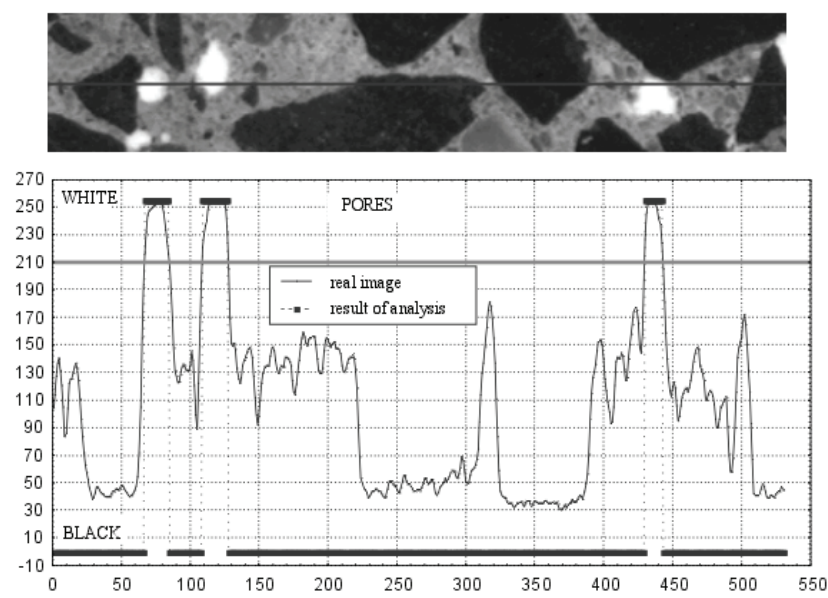

Fig. 3. Graphic interpretation of places recognized as aerial pores in basalt aggregate concrete.

Table 1 summarizes stereological analysis results by giving selected values of parameters characterizing the structure of pores which have been determined both by Method I using the FRAKTAL_Stereolog computer program and by Method II using the Image Pro Plus 4.1 image analyzer operating with a stereoscopic microscope. For the results obtained by Method I, also the standard deviation of the mean value is given. In this case, the mean value has been determined on the basis of 48 observation sites, each with an area equal to $2047.68 \mathrm{~mm}^{2}$. An observation area of dimensions of $63.2 \mathrm{~mm} \times 32.5 \mathrm{~mm}$ was adopted, making up $1 / 4$ of the area marked off on each specimen after cutting off its edges (Fig. 4). The limitation of the field subjected to analysis resulted from cutting off the side edges due to the occurrence the "wall effect" known in the concrete technology, that is, a change in the composition (structure) in the vicinity of the mould walls, associated with change in the cement paste/ aggregate proportion.

2 The designation adopted based on reference (Konkol and Prokopski, 2004). 
Both compared methods showed a variation in porosity, depending on the water-cement ratio. The concrete of a lower $W / C$, amounting to 0.44 , was more porous that the concrete with the ratio $W / C=$ 0.58 . In the case of the concrete with $W / C=0.58$, comparable values of porosity, as determined by both methods, were obtained; for the concreted with $\mathrm{W} / \mathrm{C}$ $=0.44$, on the other hand, a greater value was obtained using Method I. The difference might result from the number of specimens (images) examined and on the method of determining the value of total air content, $A$. The total air content, as examined by Method I, was determined on the basis of the planimetred pore areas, while the determination of the total air content in Method II was achieved based on the fraction of chords relative to the total length of measurement lines. The area on which measurements were made, for Method I was about 14 times as large as for Method II. Using Method I, greater values of the relative pore number, $N_{A}$, and the average pore chord, $\bar{l}$, were also obtained. At the same time, the relative pore number was determined differently for both methods, as in the case of the total air content $A$. Using Method II, larger values were only obtained for the pore specific surface, $\alpha$.

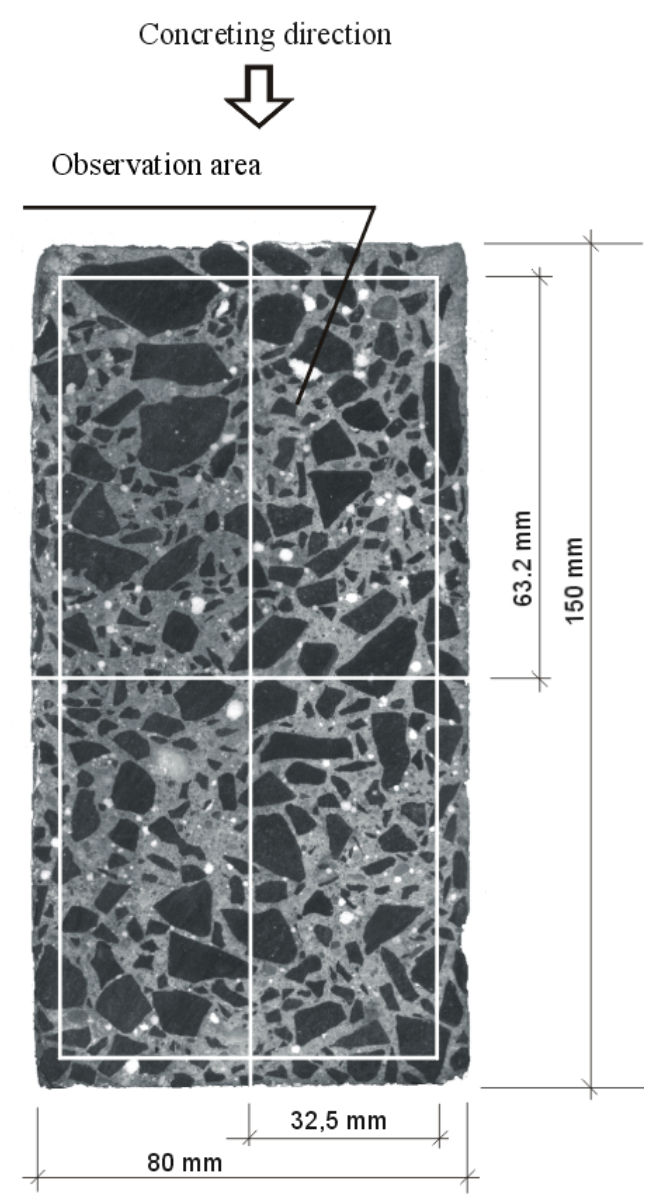

Fig. 4. Method of separating fields for examination.

Table 1. Results of the stereological analysis.

\begin{tabular}{|c|c|c|c|c|c|}
\hline \multirow{2}{*}{ No. } & \multicolumn{2}{|l|}{ Stereological parameter } & \multirow{2}{*}{$\begin{array}{c}\text { Specimen } \\
W / C\end{array}$} & \multicolumn{2}{|c|}{ Results of analysis } \\
\hline & Description & Unit & & Method I & Method II \\
\hline \multirow{2}{*}{1} & \multirow{2}{*}{ Total air content, $A$} & \multirow{2}{*}{$\%$} & 0.44 & $6.38 \pm 0.36$ & 4.29 \\
\hline & & & 0.58 & $3.53 \pm 0.18$ & 3.49 \\
\hline \multirow{2}{*}{2} & \multirow{2}{*}{ Pore specific surface, $\alpha$} & \multirow{2}{*}{$\mathrm{mm}^{-1}$} & 0.44 & $4.41 \pm 0.41$ & 10.74 \\
\hline & & & 0.58 & $5.37 \pm 0.92$ & 11.87 \\
\hline \multirow{2}{*}{3} & \multirow{2}{*}{ Observation area, $A_{z}$} & \multirow{2}{*}{$\mathrm{mm}^{2}$} & 0.44 & \multirow{2}{*}{$98592^{*}$} & 6921.02 \\
\hline & & & 0.58 & & 7261.51 \\
\hline \multirow{2}{*}{4} & \multirow{2}{*}{ Relative pore number, $N / A_{z}$} & \multirow{2}{*}{$\mathrm{mm}^{-2}$} & 0.44 & $0.083 \pm 0.001$ & 0.055 \\
\hline & & & 0.58 & $0.073 \pm 0.001$ & 0.049 \\
\hline \multirow{2}{*}{5} & \multirow{2}{*}{ Average chord length, $\bar{l}$} & \multirow{2}{*}{$\mathrm{mm}$} & 0.44 & $0.91 \pm 0.004$ & 0.372 \\
\hline & & & 0.58 & $0.77 \pm 0.007$ & 0.337 \\
\hline \multirow[b]{2}{*}{6} & \multirow{2}{*}{ Cement paste content of concrete, $p$} & \multirow{2}{*}{$\%$} & 0.44 & $25.81^{\dagger)}$ & 25.8 \\
\hline & & & 0.58 & $38.32^{\dagger)}$ & 38.3 \\
\hline \multirow{2}{*}{7} & \multirow{2}{*}{ Pore spacing factor, $\bar{L}$} & \multirow{2}{*}{$\mathrm{mm}$} & 0.44 & - & 0.47 \\
\hline & & & 0.58 & - & 0.56 \\
\hline \multirow{2}{*}{8} & \multirow{2}{*}{ Micropore content, $A_{300}$} & \multirow{2}{*}{$\%$} & 0.44 & - & 0.50 \\
\hline & & & 0.58 & - & 0.65 \\
\hline
\end{tabular}




\section{RESULTS}

Two concrete mixes of a constant ratio of cement to sand $(1: 1.76)$ and sand to basalt $(1: 1.8)$, and two water-cement ratios $(0.44$ and 0.58$)$, were prepared. The composition of the concrete mixes are given in Table 2. The total air content $A$ was determined by stereological methods using computer program FRAKTAL_Stereolog, whereas the fractal dimension $D_{B C}$ was computed using software application FRAKTAL_Wymiar $2 D^{3}$ ). The images subjected to stereological and fractal analyses were sectioned off from the concrete specimen surfaces formed by cutting bars used in fracture toughness tests performed according to Mode I of fracture (tension at bending), in confor-mance to the guidelines of the RILEM draft reco-mmendations (RILEM Report 89FMT). From the specimen surface with $80 \times 150 \mathrm{~mm}$ dimensions, in order to eliminate the errors resulting from the ,wall effect”, an area of $64.8 \times 126.7 \mathrm{~mm}$ was chosen for analysis.

Prepared surfaces were scanned at resolution of 400 dpi, resulting in a bitmap of $1021 \times 1997$ pix $^{2}$. Then the specimen surface image (Fig. 5) was converted to a binary image, in which the black elements were pores (Fig. 6). The stereological analysis was performed in FRAKTAL_Stereolog program applying basic morphological and punctual transformations. The applied procedure allowed pores of sizes from $6 \mathrm{pix}^{2}\left(0.0242 \mathrm{~mm}^{2}\right)$ to be detected. The description of successive steps in image preparation and performed computation are listed as a flow chart (Fig. 7).

In this analysis, pixels of a grayscale value from 210 to 255 (Step 1 of analysis) were deemed pores; in a grayscale image, the pixel of a value of 0 is black in colour, and the pixel of a value of 255 is white in colour. In Step 2 of analysis the grayscale (original) image was converted to a binary image (with black pores in the white background). The operation of pouring the pores (Step 6) involved the removal of the noise inside the pores while the aim of noise elimination (Step 7) was to remove the noise in the form of isolated pixels and to smooth out the pore edges. This was accomplished by performing the operation of reconstruction with single erosion.

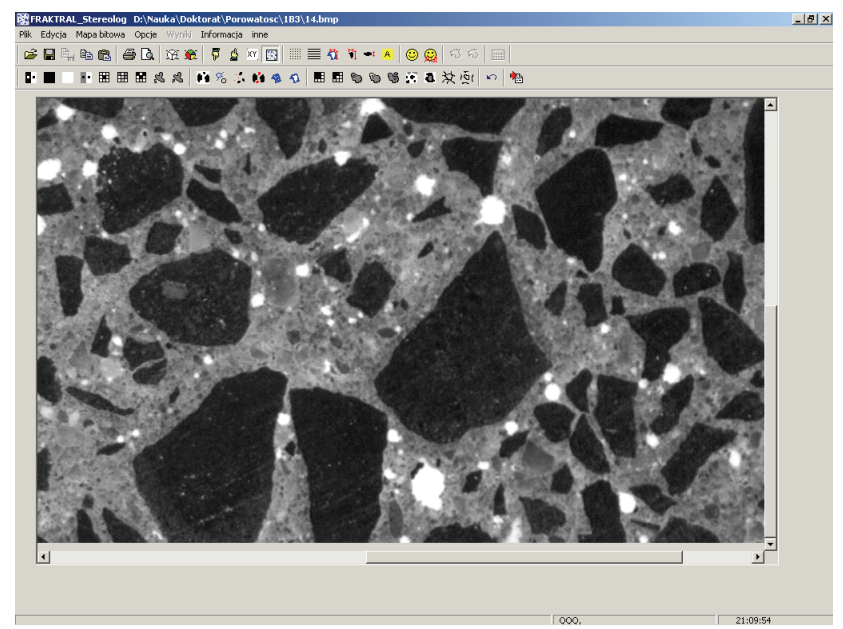

Fig. 5. The image of the surface of specimen If.

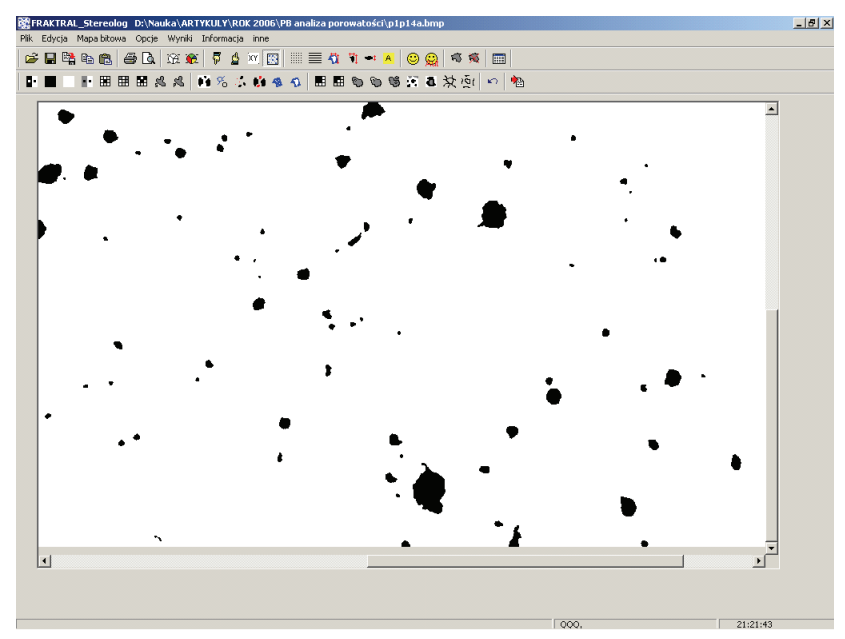

Fig. 6. The image of pores after Step 7 of analysis (specimen If).

The results of stereological and fractal measurements are shown in Table 3 .

Table 2. Composition of concrete mixes.

\begin{tabular}{|c|c|c|c|c|}
\hline \multirow{2}{*}{ Serie $(W / C)$} & \multicolumn{4}{|c|}{ Composition of concrete mixes $\left[\mathrm{kg} / \mathrm{m}^{3}\right]$} \\
\cline { 2 - 5 } & Cement & Sand & Basalt & Water \\
\hline Serie I $(0.44)$ & 396.1 & 698.4 & 1260.6 & 174.0 \\
\hline Serie II $(0.58)$ & 375.1 & 661.3 & 1193.7 & 217.8 \\
\hline
\end{tabular}

3 Konkol J.: FRAKTAL_Wymiar2D. A computer program. 


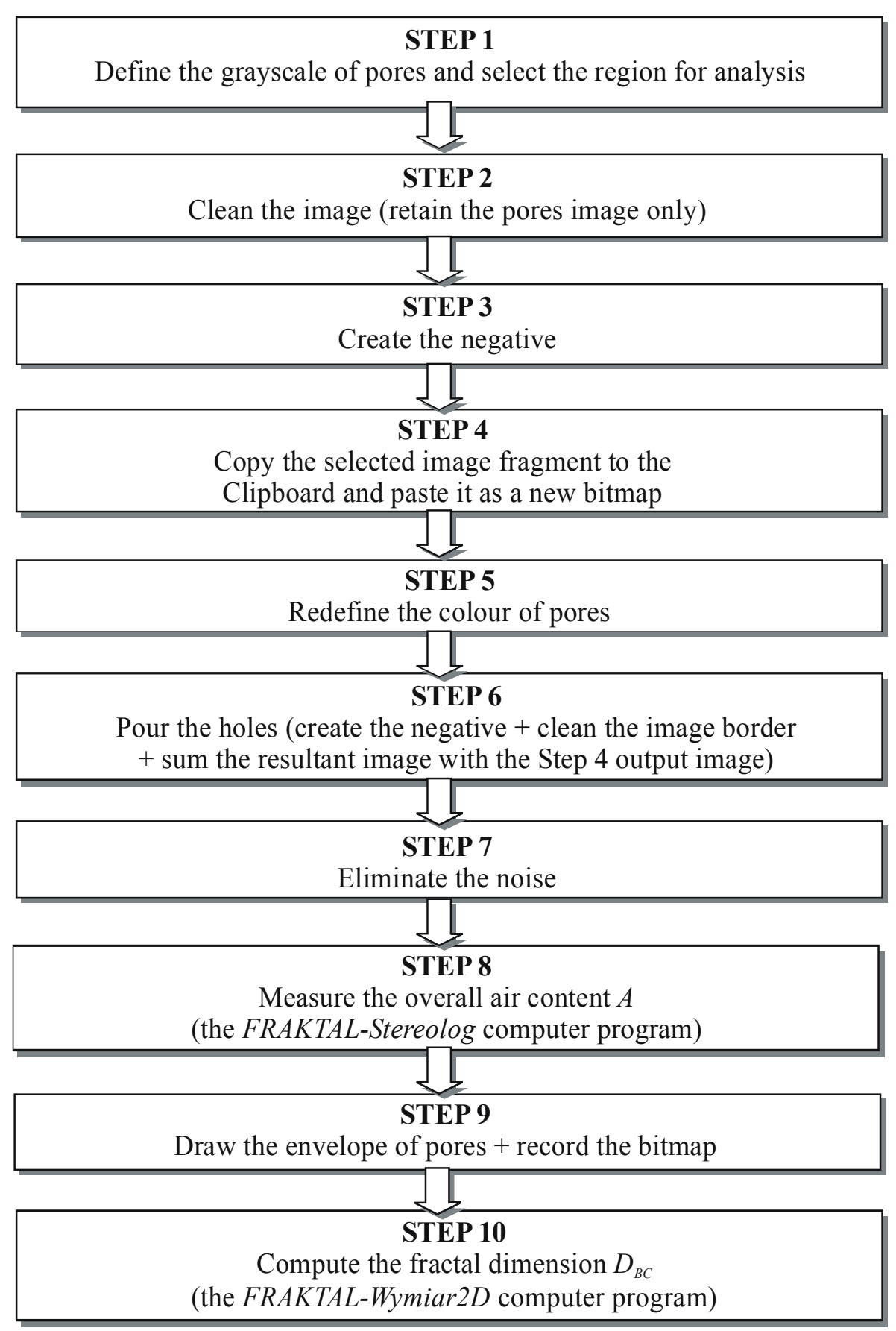

Fig. 7. Flow chart for the determination of the total air content $A$ and the fractal dimension $D_{B C \text {. }}$

The relative pores number was computed according to the formula below:

$$
N_{A}=\frac{N}{A_{Z}},
$$

where

$N$ - the total number of pores in the specimen image;

$A_{Z}$ - the size of the observed image.
The fractal dimension $D_{B C}$ was determined from the slope of the straight line of the relationship of $\log$ $N(s)$ versus $\log (s)$, where the step $s$ varied from 1 do 20 pixels, that is from 0.064 to $1.272 \mathrm{~mm}$. For each step $s$, the specimen surface image was covered with a grid of squares of the side equal to that step, and then the counting of the squares which included pore edges was made. An example of the image of a specimen surface covered with a grid of squares is shown in Fig. 8. 


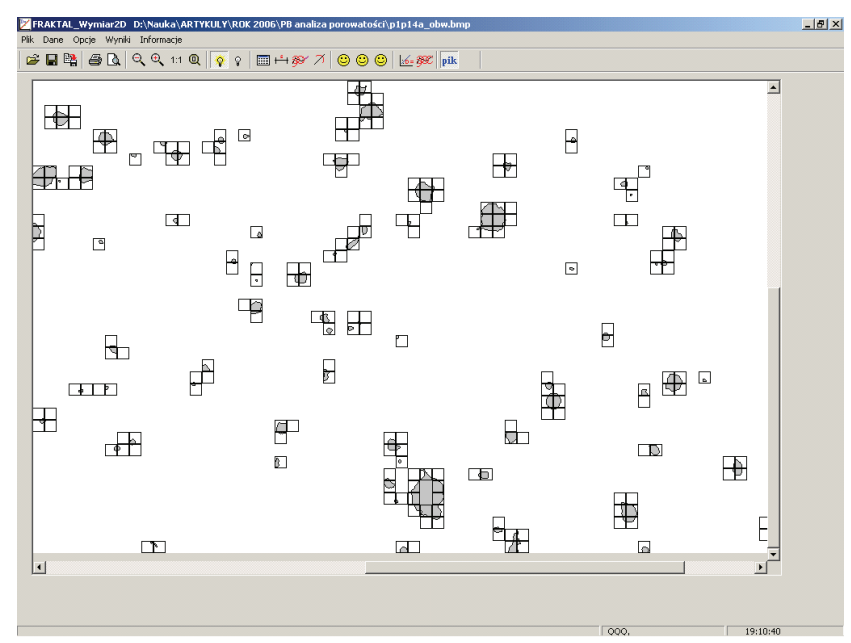

Fig. 8. Visible edges of pores with the grid of squares covering them (specimen If).

\section{DISCUSSION}

The obtained values of the fractal dimension $D_{B C}$ for the specimens of Series I and II indicate a different distribution of pores in these specimens. This is confirmed by the sample images of pores on the specimen surface, as shown in Fig. 9. The greatest total porosity, as well as a greater number of fine pores, were found in the specimens of Series I (see the values of $N, N_{A}$ and $A$, Table 3). While larger pores are visible in both series, the pores in the specimens of Series I, however, have irregular shapes, which indicates that these are technological pores formed as a result of the hindered consolidation of mixes of this concrete. The greater number of smaller pores, as well as the occurrence of pores with irregular shapes, resulted in larger values of the fractal dimension $D_{B C}$ of pores obtained in the concrete of Series I compared to the concrete of Series II.

In order to determine the relationship between the fractal dimension $D_{B C}$ and the computed para-meters, we used statistical analysis on the obtained results.

Two models of multiple regression were proposed:

1. the dependence of the fractal dimension $D_{B C}$ on the relative number of pore cross-sections, $N_{A}$, (or possibly the number of pore cross-sections, $N$ ) and the total air content, $A$,

2. the dependence of the fractal dimension $D_{B C}$ on the relative number of pore cross-sections, $N_{A}$, and the water-cement ratio, $W / C$, of the following forms:

$$
\begin{aligned}
& D_{B C}=(1111.378+0.421 \cdot N+29.772 \cdot A) \cdot 10^{-3} \\
& D_{B C}=\left(1111.378+34.567 \cdot N_{A}+29.772 \cdot A\right) \cdot 10^{-3}
\end{aligned}
$$

and

$D_{B C}=(1409.78+0.462 \cdot N-435.968 \cdot W / C) \cdot 10^{-3}$

$D_{B C}=\left(1409.78+37.933 \cdot N_{A}-435.968 \cdot W / C\right) \cdot 10^{-3}$

\begin{tabular}{|c|c|c|c|c|c|}
\hline \multirow[b]{2}{*}{ No. } & \multirow[b]{2}{*}{ Specimen } & \multirow[b]{2}{*}{$W / C$} & \multicolumn{2}{|c|}{ Stereological parameters } & \multirow[b]{2}{*}{ Fractal dimension $D_{B C}$} \\
\hline & & & $\begin{array}{l}\text { Number of pores, } N \\
\text { (relative pores } \\
\text { number } N_{A} \text { ) }\end{array}$ & $\begin{array}{c}\text { Total air content } A \text {, } \\
\%\end{array}$ & \\
\hline 1 & I a & 0.44 & $303(3.691)$ & 3.12 & 1.3465 \\
\hline 2 & $\mathrm{Ib}$ & 0.44 & $259(3.155)$ & 3.76 & 1.3571 \\
\hline 3 & $\mathrm{I} \mathrm{c}$ & 0.44 & $421(5.128)$ & 4.11 & 1.4018 \\
\hline 4 & I d & 0.44 & $344(4.190)$ & 5.06 & 1.3790 \\
\hline 5 & $\mathrm{Ie}$ & 0.44 & $218(2.655)$ & 3.57 & 1.3269 \\
\hline 6 & If & 0.44 & $273(3.325)$ & 3.76 & 1.3383 \\
\hline \multicolumn{3}{|c|}{ Mean values } & 303 (3.691) & 3.90 & 1.3583 \\
\hline 7 & II a & 0.58 & $123(1.498)$ & 1.98 & 1.2102 \\
\hline 8 & II b & 0.58 & $112(1.364)$ & 1.50 & 1.1902 \\
\hline 9 & II c & 0.58 & $151(1.839)$ & 1.73 & 1.2129 \\
\hline 10 & II d & 0.58 & $192(2.339)$ & 1.81 & 1.2574 \\
\hline 11 & II e & 0.58 & $185(2.253)$ & 1.66 & 1.2399 \\
\hline 12 & II $\mathrm{f}$ & 0.58 & $141(1.717)$ & 1.52 & 1.2107 \\
\hline 13 & II $g$ & 0.58 & $185(2.253)$ & 2.60 & 1.2781 \\
\hline \multicolumn{3}{|c|}{ Mean values } & $155.6(1.895)$ & 1.83 & 1.2285 \\
\hline \multicolumn{6}{|c|}{ Additional result } \\
\hline 14 & I g & 0.44 & $280(3.410)$ & 3.23 & 1.3324 \\
\hline
\end{tabular}

Table 3. Results of the stereological and fractal analyses. 
For both series of concretes, the correlation coefficient of the adopted models of multiple regression amounted to $R=0.978$, and the obtained relationships, due to the critical value of the level of significance $(p<<0.5)$, can be regarded and statistically significant. The computed standardized (beta) regression coefficients were:

- for the first model of multiple regression

$$
\begin{aligned}
& \text { - } \quad \text { for } \mathrm{N}_{\mathrm{A}}(\text { or } \mathrm{N}) \quad 0.532, \text { at } \mathrm{p} \approx 0.002, \\
& \text { - } \quad \text { for } \mathrm{A} \quad 0.483 \text {, at } \mathrm{p} \approx 0.004,
\end{aligned}
$$

- for the second model

$$
\begin{array}{ll}
\text { - } \quad \text { for } \mathrm{N}_{\mathrm{A}}(\text { or } \mathrm{N}) & 0.583, \text { at } \mathrm{p} \approx 0.0006 \\
\text { - } \quad \text { for } \mathrm{W} / \mathrm{C} & -0.44, \text { at } \mathrm{p} \approx 0.0042
\end{array}
$$

The beta coefficients indicate the relative effects, which each of the independent variables contributes to the prediction of the dependent variable $D_{B C}$. For both models, the most important predictor of the fractal dimension $D_{B C}$ is the relative number of pore cross-sections, $N_{A}$. At the same time, the levels of significance, $p$, show that all of the independent variables are statistically significant. The beta coefficient, as well as the regression function coefficient, related to the relative number of pore cross-sections,
$N_{A}$, (or the number of pores, $N$ ), and the total air content, $A$, are positive, which means that the greater the values of $N_{A}$ (or $N$ ) or $A$, the greater is the fractal dimension $D_{B C}$. The coefficients related to the watercement ratio, on the other hand, are negative, which means that the greater water-cement ratio, $W / C$, the lesser smaller is the fractal dimension, $D_{B C}$. In the light of concrete technology such dependency is obvious. Concretes with higher water-cement $W / C$ ratio are characterized by a heavy consistency and better workability, which in turn results in better density of the concrete mix. Making concrete mixes with lower water-cement $W / C$ ratios thicker may be more difficult which results in creation, in the structure of such concrete, pores that are larger and more irregular (Fig. 1d), which has significant effect on the overall porosity, as well as the value of the fractal dimension (Table 3).

The relationship of the observed values of $D_{B C}$ versus the predicted values of $D_{B C}$, together with confidence intervals, are shown in Figs. 10 and 11. The obtained results have been grouped by water-cement ratio, which is marked in the figures in the form of the two regions of results, obtained for the concrete with $W / C$ $=0.44$ and $W / C=0.58$, respectively.

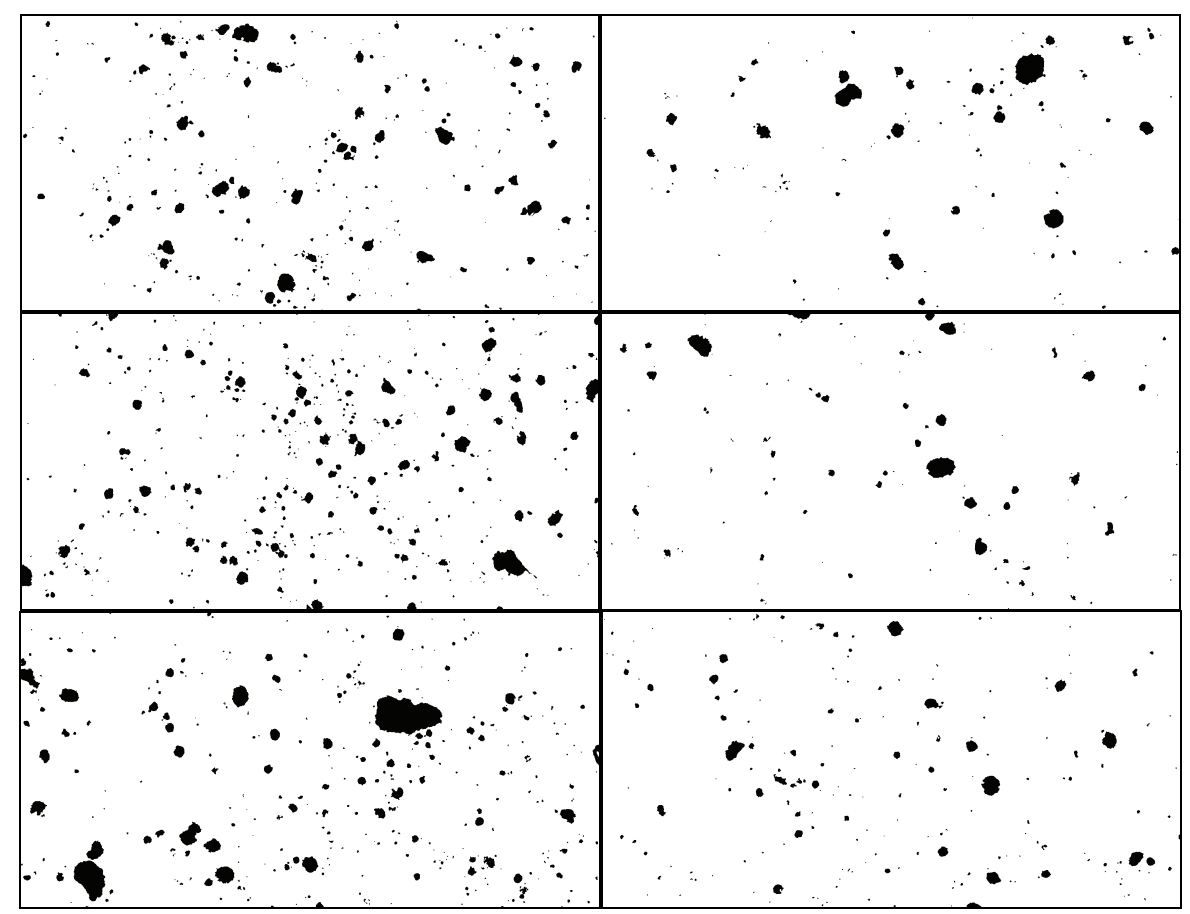

Fig. 9. Example images of the pores distribution of in specimens: I a and II a (at the top), I $c$ and II $b$ (in the middle), I d and II d (at the bottom). 


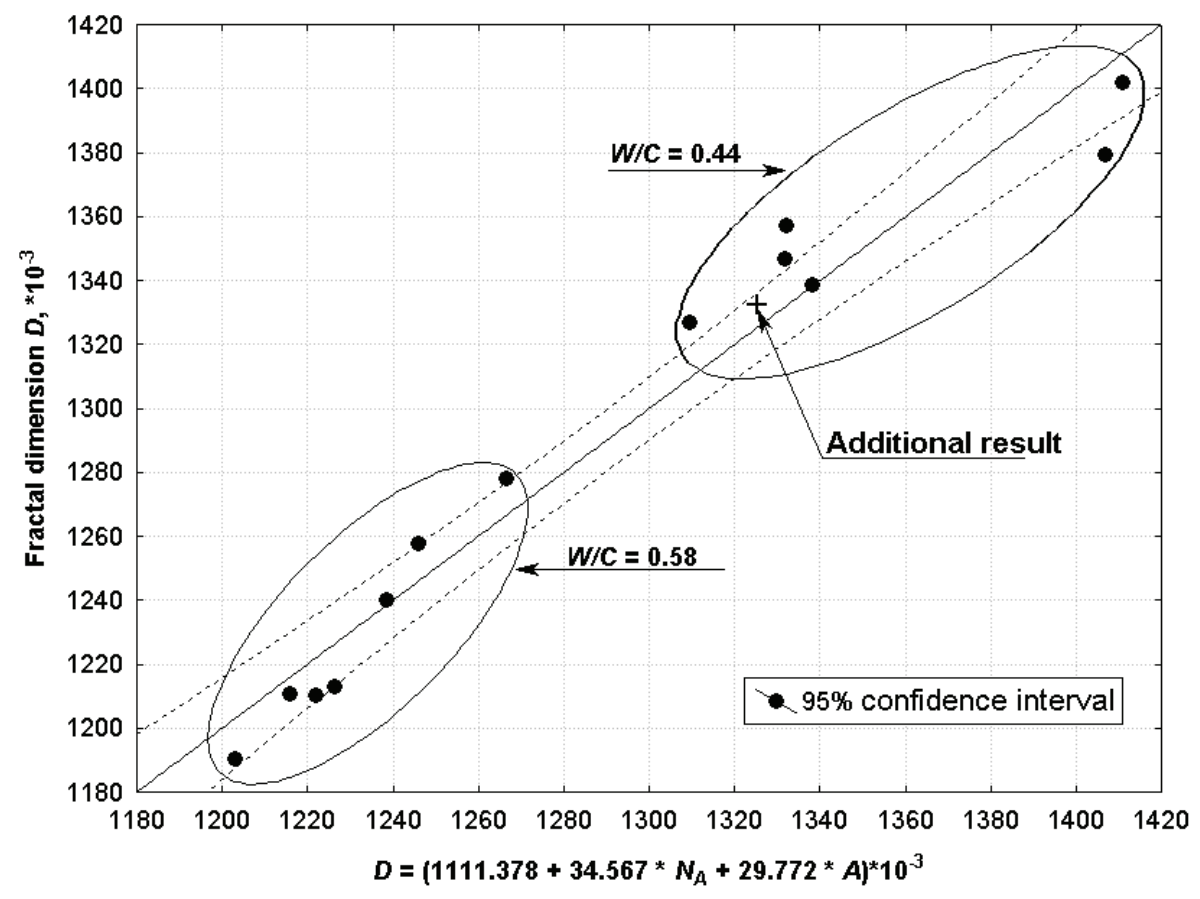

Fig. 10. Fractal dimension values as observed against those predicted for Model I of multiple regression.

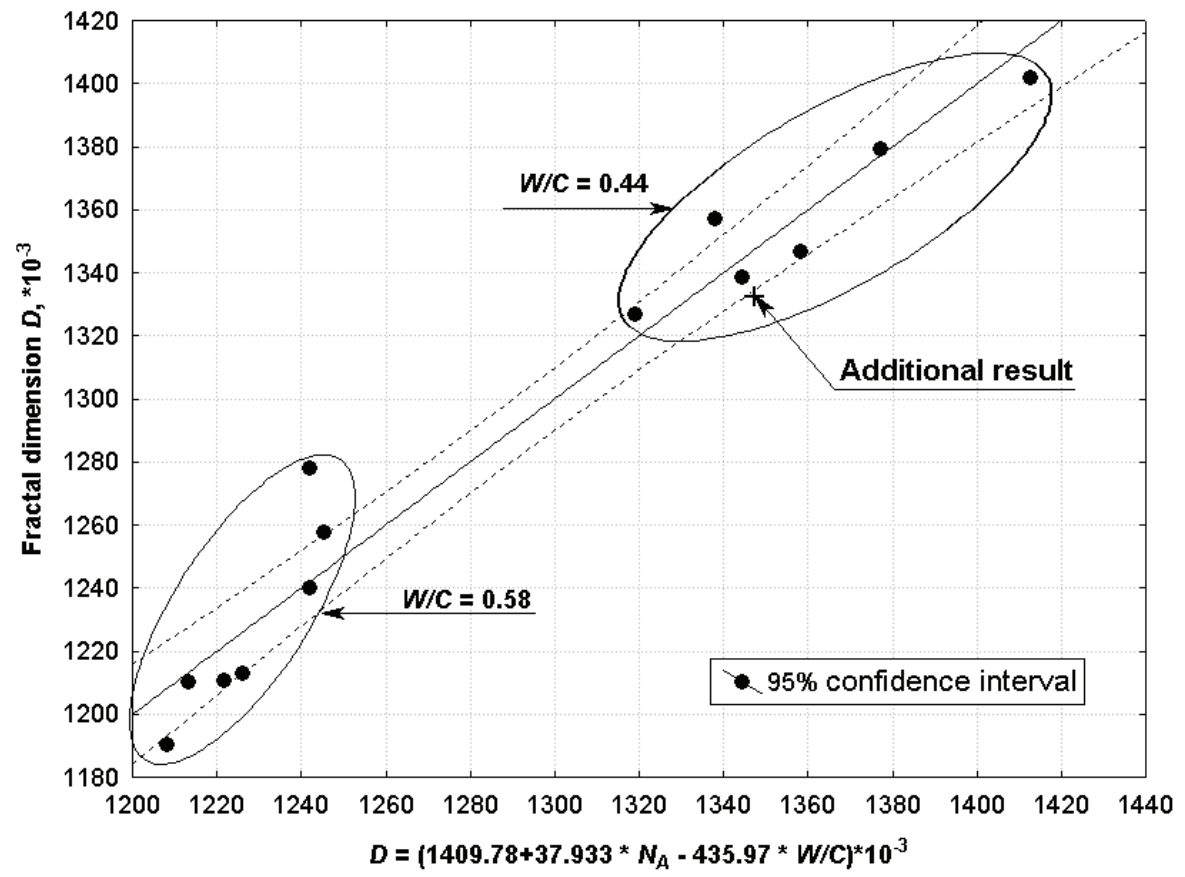

Fig. 11. Fractal dimension values as observed against those predicted for Model II of multiple regression.

In order to verify the obtained regression models, an additional specimen of concrete from Series I was prepared, and the results obtained on its basis were plotted in Figs. 10 and 11. The result of $D_{B C}$, obtained in this case on the basis of Model I of multiple regression, is underestimated by about $0.52 \%$ in relation to the actual result, while that obtained on the basis of Model II is overestimated by about $1.12 \%$. In the case 
of Model I, the additional result lies within the confidence interval, whereas for Model II it lies slightly outside it. The position of both additional points indicates the conformance of the obtained additional results with relationships (4) and (5).

\section{CONCLUSION}

In this work we have presented the application of a new method of assessment of concrete porosity diversification using the fractal dimension $D_{B C}$ as an additional parameter characterizing the macro-pore structure with stereological methods.

The analysis concerning the porosity of concretes was performed on the flat cross-sections of concrete specimens using image analysis. By using basic morphological and punctual transformations and the proper preparation of specimens, a binary image of macro-pores in the hardened concrete was obtained from the real image of the specimen. The stage of preparation of the specimen and the image is one of the most difficult tasks preceding the stereological analysis. The image must be prepared so as to eliminate the possibility of noise resulting from an erroneous assignment of other concrete structure elements, e.g. pores.

The advantage of the stereological methods over other methods of determining the morphology of pores in concrete is the method of making measurements. The stereological analysis is carried out on cross-sections, where pores, their shapes and distribution are visible. The standardization of these measu-rements and their commonness, both in Europe (EN 480-11) and in the USA (ASTM C 45798), provide evidence for high reliability of examination results.

The method of describing macro-pores with the fractal dimension $D_{B C}$, as presented in this article, expands the possibilities of differentiating the porosities of concretes (as well as other materials), while allowing for both the total porosity and the number and shape of pores.

The performed examination of concretes has show the existence of a statistically significant correlation (at a significance level of $p=0.05$ ) between the fractal dimension $D_{B C}$, as determined by the box method, and the relative number of pore cross-sections $N_{A}$ (or the number of pores $N$ ) and the total air content $A$, and between the fractal dimension $D_{B C}$ and the relative number of pore cross-sections $N_{A}$ and the water-cement ratio $W / C$.
The application of the fractal dimension $D_{B C}$ to the description of porosity has proved to be an efficient method, and the obtained results and correlations indicate full usefulness of the applied method for the assessment of diversification of the porosity of set concrete.

\section{REFERENCES}

Aligizaki KK, Cady PC (1999). Air content size distribution of air voids in hardened cement pastes using the section-analysis method. Cem Concr Res 29:273-80.

ASTM C 457 - 98 (1998). Standard test method for microscopical determination of parameters of the air-void system in hardened concrete, 228-41.

Carpinteri A, Chiaia B, Invernizzi S (1999). Three-dimensional fractal analysis of concrete fracture at the mesolevel. Theoretical and applied fracture mechanics 31 : 163-72.

Carpinteri A, Chiaia B, Nemati KM (1997). Complex fracture energy dissipation in concrete under different loading conditions. Mech Mat 6:93-108.

Carpinteri A, Spagnoli A (2004). A fractal analysis of size effect on fatigue crack growth. Int J Fatigue 26:125-133.

Carpinteri A, Spagnoli A, Vantadori S (2010). A multifractal analysis of fatigue crack growth and its application to concrete. Eng Fract Mech 77:974-84.

Chiaia B, van Mier JGM, Vervuurt A (1998). Crack growth mechanisms in four different concrete: Microscopic observations and fractal analysis. Cem Concr Res 28(1): $103-14$

Cook RA, Hover KC (1999). Mercury porosimetry of hardened cement pastes. Cem Concr Res 29:933-43.

Daian JF (1988). Condensation and isothermal water transfer in cement mortar. Part I - Pore size distribution, equilibrium, water condensation and imbibition. Transport in Porous Media 3:563-89.

Diamond S (2000). Mercury porosimetry. An inappropriate method for the measurement of pore size distributions in cement-based materials - review. Cem Concr Res 30: $1517-25$.

Elsen J (2001). Automated air void analysis on hardened concrete. Results of a European intercomparison testing program. Cem Concr Res 31:1027-31.

Fracture Mechanics Test Methods for Concrete. RILEM Report 89-FMT, edited by S.P. Shah and A. Carpinteri, Chapman and Hall 1991.

Gregg SJ, Sing KSW (1982). Adsorption, Surface Area and Porosity. $2^{\text {nd }}$ ed. London: Academic Press.

Hu J, Stroeven P (2003). Application of image analysis to assessing critical pore size for permeability prediction of cement paste. Image Anal Stereol 22:97-103.

Ji X, Chan SYN, Feng N (1997). Fractal model for simulating the space-filling process of cement hydrates and fractal dimensions of pore structure of cementbased materials. Cem Concr Res 27(11):1691-9. 
Konkol J, Prokopski G (2004). Analysis of the fracture surface morphology of concrete by the method of vertical section. Computers and Concrete 1(4):389-400.

Lange DA, Jennings HM, Shah SP (1994). Image analysis techniques for characterization of pore structure of cement-based materials. Cem Concr Res 24(5):841-53.

Li L, Chan P, Zollinger DG, Lytton RL (1993). Quantitative analysis of aggregate shape based on fractals. ACI Materials Journal 90(4):357-65.

Mandelbrot BB (1977). Fractals. Form, chance and dimension. San Francisco: Freeman.

Powers TC (1949). The air requirement of frost-resistant concrete. Proceedings, Highway Research Board 29: 184-202.

Prokopski G, Konkol J (2005). The fractal analysis of the fracture surface of concretes made from different coarse aggregates. Computers and Concrete 2(3):239-48.

Ringot E, Bascoul A (2001). About the analysis of microcracking in concrete. Cem Conc Comp 23:261-6.
Stroeven P, Guo Z (2006). Modern routes to explore concrete's complex pore space. Image Anal Stereol 25: 75-86.

Tanaka K, Kurumisawa K (2002). Development of technique for observing pores in hardened cement paste. Cem Concr Res 32:1435-41.

Winslow DN (1985). The fractal nature of the surface of cement paste. Cem Concr Res 15:817-24.

Yan A, Wu K, Zhang X (2002). A quantitative study on the surface crack pattern of concrete with high content of steel fiber. Cem Concr Res 32:1371-5.

Załocha D (2002). Image analysis as a tool for estimation of air-void characteristics in hardened concrete: example of application and accuracy studies. A.M.A.S. Workshop - SIAIC'02. Structural Image Analysis in Investigation of Concrete. Conference Warsaw (edited by Kasperkiewicz J, Brandt AM), October 21-23, 23957. 